\title{
Experimental results of an rf gun and the generation of a multibunch beam
}

\author{
Abhay Deshpande, ${ }^{1, *}$ Sakae Araki, ${ }^{2}$ Masafumi Fukuda, ${ }^{2}$ Kazuyuki Sakaue, ${ }^{3}$ \\ Nobuhiro Terunuma, ${ }^{2}$ Junji Urakawa, ${ }^{2}$ and Masakazu Washio ${ }^{3}$ \\ ${ }^{1}$ Department of Accelerator Science, School of High Energy Accelerator Science, Graduate University for Advanced Studies, \\ Shonan International Village, Hayama, Miura, Kanagawa 240-0193, Japan \\ ${ }^{2}$ High Energy Accelerator Research Organization, 1-1 Oho, Tsukuba, Ibaraki 305-0801, Japan \\ ${ }^{3}$ Waseda University, Shinjuku-ku, Tokyo 169-8555, Japan
}

(Received 2 July 2010; published 2 June 2011)

\begin{abstract}
At the laser undulator compact x-ray source at KEK, our group has designed and built a new rf gun with a larger mode separation of $8.6 \mathrm{MHz}$ and higher $Q$ value than earlier guns. This paper presents details on the design and fabrication, low-power measurements, and tuning procedures followed in the fabrication of the gun cavity. We also give a detailed account of experiments performed with this gun and present measurement results. We have successfully generated 100 bunches per train with a $50 \mathrm{nC}$ charge at $41 \mathrm{MeV}$ and 300 bunches per train with a $160 \mathrm{nC}$ charge at $5 \mathrm{MeV}$, with low peak-to-peak energy difference. In the future, we plan to go up to a $0.5 \mathrm{nC}$ charge per bunch in an 8000-bunch train at low energy and a $2 \mathrm{nC}$ charge per bunch in a 100-bunch train at high energy. This will make it possible to have higher charge available for laser-beam collisions, thus enabling the generation of high flux soft $\mathrm{x}$ rays using the low-energy electron beam and approximately $30 \mathrm{keV}$ x rays using the high-energy electron beam.
\end{abstract}

PACS numbers: 29.25.Bx, 29.20.Ej, 29.27.Fh, 29.27.Ac

\section{INTRODUCTION}

\section{A. rf gun and accelerator}

Our group at KEK has designed and developed a compact, S-band Laser Undulator Compact X-ray Source (LUCX) [1,2]. The LUCX setup is shown in Fig. 1 below. This test bench has an rf photocathode gun to inject a lowemittance beam with a bunch spacing of $2.8 \mathrm{~ns}$ in a $3 \mathrm{~m}$ long S-band traveling wave, constant-gradient linear accelerator (linac). When the beam is injected, the linac accelerates the bunches to $40 \mathrm{MeV}$ energy with a peak-to-peak energy difference of less than $0.7 \%$ using a $\Delta T$ compensation technique [3]. This high-energy beam then goes through a quadrupole doublet to reach a beam size of the order of $40 \mu \mathrm{m}$ in the interaction region. The beam collides with a pulsed laser inside a supercavity and produces intense $\mathrm{x}$ rays via the mechanism of inverse Compton scattering. Our group recently used this setup to produce $30 \mathrm{keV} x$ rays with a flux of $1.2 \times 10^{5}$ photons per second [4]. To increase the flux further, we began working on various systems to optimize the parameters and achieve a higher flux.

Changes were initiated in the rf gun and the accelerator to improve the performance. Our group designed a new rf photocathode gun cavity to increase the mode separation and the quality factor [5-7]. The cavity profile of the rf gun is changed from the Brookhaven National Laboratory

\footnotetext{
*abhay@ post.kek.jp; astroabhay1@yahoo.com
}

Published by the American Physical Society under the terms of the Creative Commons Attribution 3.0 License. Further distribution of this work must maintain attribution to the author(s) and the published article's title, journal citation, and DOI.
(BNL) type IV to a new curved-surface type as shown in the next section. The mode separation between the operating $\pi$ mode and zero mode was increased from $3.5 \mathrm{MHz}$ in the old gun to $8.6 \mathrm{MHz}$ in the new gun. Once the new rf gun was fabricated and installed, we measured various parameters and found that its energy spread was more stable for phase variations than the old gun with smaller mode separation. Simulations done by Linac Coherent Light Source (LCLS) and other groups have demonstrated this stability [8-10], and our measurements confirm the predictions.

One of the main aims of our work is to generate long bunch trains. Specifically, we seek to produce a very long pulse train with a bunch spacing of $2.8 \mathrm{~ns}$ and accelerate the train using a normal conducting accelerator. Earlier we demonstrated a 100 bunch beam with $2.8 \mathrm{~ns}$ spacing and a $50 \mathrm{nC}$ charge at $40 \mathrm{MeV}$ beam energy. We then worked out a new scheme, as proposed earlier, to make a series of long bunch trains with a higher charge per train [11]. We replaced the linac in our setup with a drift tube and achieved a $5 \mathrm{MeV}$ beam with a $160 \mathrm{nC}$ charge in 300 bunches with a peak-to-peak energy difference of less than $0.85 \%$. Encouraged by the results, we are now building a 3.5 cell $\mathrm{rf}$ gun capable of attaining $10 \mathrm{MeV}$ beam energy with 8000 bunches per train and $0.5 \mathrm{nC}$ charge per bunch, mainly for application in medicine and other fields. This paper presents the experimental results of the modeseparated rf gun and the generation of a 300 bunch train.

To increase the flux of photons coming from an inverse Compton source like LUCX, various options are available. The designer can increase the charge per bunch to a high value and increase the repetition rate to a moderately high value to increase the number of electrons colliding with the 


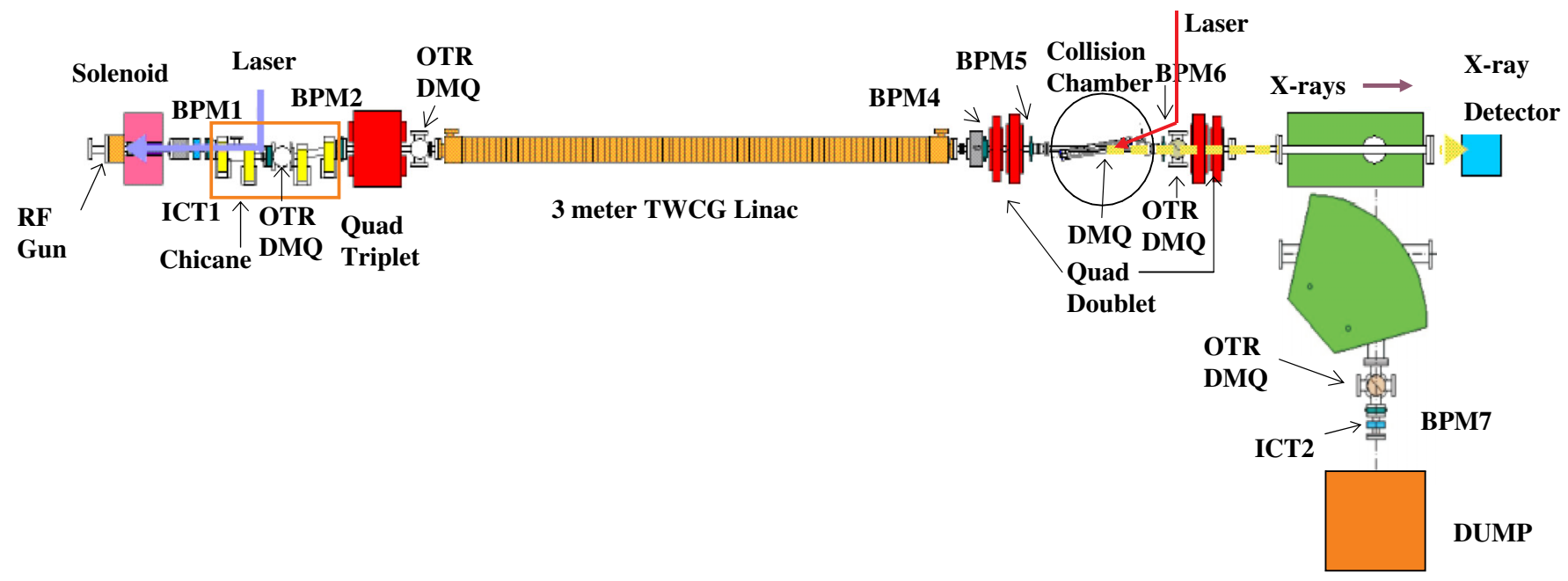

FIG. 1. Setup of the LUCX test facility. The rf gun is installed at the left end and the dump is positioned at the bottom right. The collision chamber is the interaction region where the electron beam collides with the laser pulse to produce $\mathrm{x}$ rays via inverse Compton scattering. Two independent laser systems are used, one to produce electron bunches in the rf gun and one to produce laser pulses to collide with the bunches.

laser photons per second. In this approach the main problem is that the emittance of beam increases with charge which may result in degrading effects after collision. The other option is to build a source with low charge per bunch and very high repetition rate, for example $200 \mathrm{~Hz}$ or above. This ensures that the emittance is low. The main problem in this approach is the difficulty to sustain high repetition rate operations. The heat dissipated is very high in such a case and the drift in parameters due to temperature is prominent. Further, it is tough to get high repetition rate components for laser system and the cost of laser system becomes very high. A simpler option, therefore, is to go for low charge per bunch with many bunches per train and operate at moderate repetition rates. In this approach, the main technological problem is to achieve the low bunch-to-bunch energy difference by proper beamloading compensation. However, once this technology is achieved, the designer gets low-emittance beam and the large number of bunches per train ensures that the charge available per unit time for collision is sufficiently high to get a large flux after collisions with laser pulses.

\section{B. Laser system for the rf gun}

The seed laser is a neodymium vanadate (Nd: YVO4) mode-locked oscillator with a $357 \mathrm{MHz}$ frequency. The average power is $7 \mathrm{~W}$ at $1064 \mathrm{~nm}$ wavelength and pulse duration of $7 \mathrm{ps}$ (FWHM). The infrared pulses produced at $2.8 \mathrm{~ns}$ (corresponding to $357 \mathrm{MHz}$ ) pass through a Faraday isolator into a Pockels cell. The Pockels cell chooses the number of pulses from within a range of 1 pulse to 8000 pulses for the operation. The selected pulses pass through a double pass amplifier, then through another Pockels cell to enhance the contrast ratio. Then they pass again through an amplifier. The high-power pulses are then passed through nonlinear optics beta barium borate crystals to produce the fourth harmonic. The final outcome is a $266 \mathrm{~nm}$ ultraviolet (UV) laser with a (FWHM) pulse length of 12.5 ps.

\section{DESIGN, FABRICATION, AND CONDITIONING OF THE RF GUN}

\section{A. The rf gun structure}

The rf gun design was changed to increase the mode separation to $8.6 \mathrm{MHz}$ while also increasing the $Q$ and shunt impedance. The advantage of the larger mode separation is an increase in the stability of the energy spread over phase variations. To attain our target of generating 8000 bunches per train with $2.8 \mathrm{~ns}$ spacing, we will require a long pulse-width klystron. In the near future, we will install a new klystron with a pulse width of $24 \mu \mathrm{s}$. The higher mode separation will help to control and minimize the increase in zero mode effects and other degrading effects resulting from the use of this longer pulse-width klystron.

The structure of the original LUCX rf gun (hereinafter, the "old gun") was close to the BNL structure. Our main modification at KEK, after years of experience, was to replace the Helicoflex seal joint with a brazing joint. Another major change was to introduce the new "deformation tuners" in the tuner region [12]. These tuners do not penetrate the gun cavity, but maintain a wall thickness of about $2 \mathrm{~mm}$. A screw-type mechanism is used to move the tuner, and with it the cavity wall, up or down, and thereby change the cavity frequency. Each cell has four diametrically opposed tuners, and the cavity frequency can be changed up to $\pm 1 \mathrm{MHz}$. The original LUCX rf gun cavity had a mode separation of $3.52 \mathrm{MHz}$ and a field balance $\left(E_{\text {half }} / E_{\text {full }}\right)$ of 1.30 . Our target was to increase the separation to $8.6 \mathrm{MHz}$. 
The mode separation was increased by increasing the coupling between the cells through two adjustments, namely, increasing the iris diameter and reducing the length of the drift tube between the half cell and full cell. We decided to maintain a field balance of 1.0 and selected an $8.6 \mathrm{MHz}$ target for final mode separation, after considering various fabrication restrictions and tuning conditions. Figures 2 and 3 below show the old and new rf gun profiles, as predicted by SUPER FISH [13]. Table I below shows the comparative parameters for the guns developed.

One of the first challenges we faced in fabrication was the curved internal profile of the cavity. We had two options for the machining of this profile. The first option, one frequently practiced, was to cut open the cavity along the center of the rf slot and turn it uniformly in two halves to make a full cell. We decided not to use this option, as it would require a complicated brazing procedure at the rf

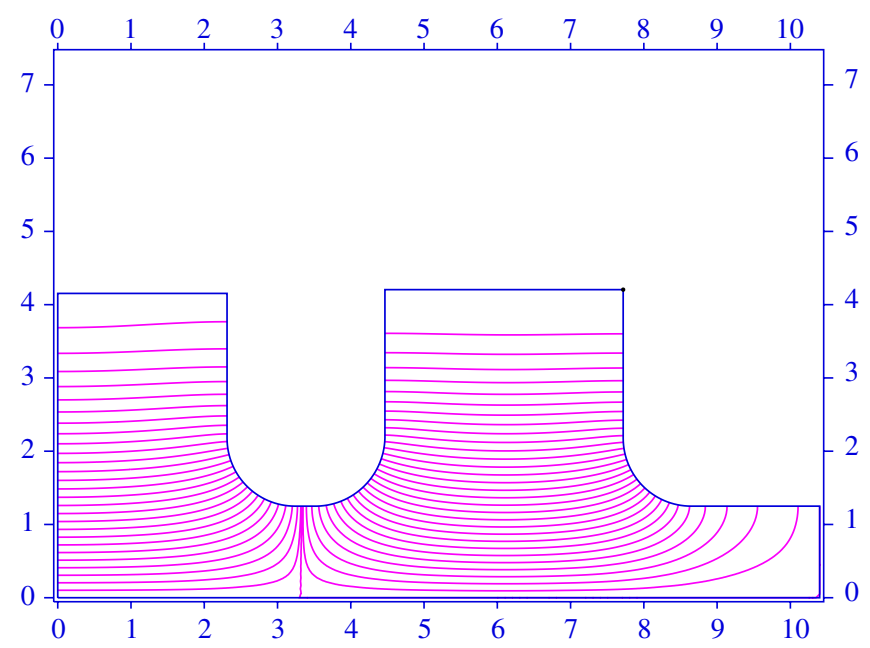

FIG. 2. Profile of the old rf gun. The old gun was close to the BNL Type IV gun.

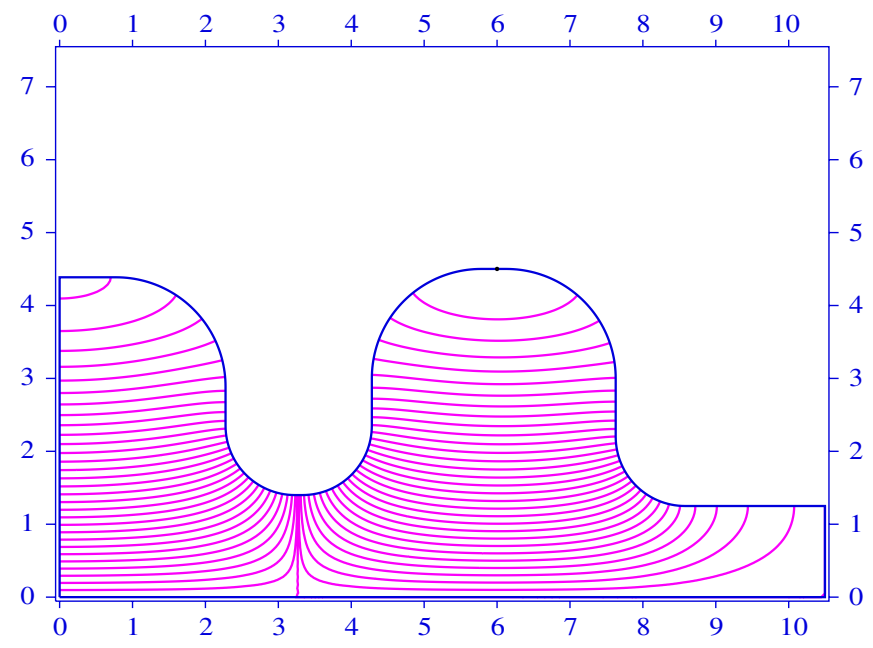

FIG. 3. Profile of the new rf gun. The iris diameter is increased to increase the mode separation.
TABLE I. Measurement results for the new and old $\mathrm{rf}$ guns.

\begin{tabular}{lcccc}
\hline \hline & \multirow{2}{*}{ Simulated } & \multicolumn{2}{c}{ Measured } & \\
& New Gun & New Gun & Old Gun & Unit \\
\hline Frequency & 2855.64 & 2855.61 & 2855.74 & $\mathrm{MHz}$ \\
Mode separation & 8.67 & 8.63 & 3.52 & $\mathrm{MHz}$ \\
Field balance & 1.0 & 0.98 & 1.3 & \\
$Q$ & 18000 & 15000 & 7900 & \\
Coupling $\beta$ & 1.0 & 1.0 & 0.6 & \\
\hline \hline
\end{tabular}

slot joint. The second option, the option we chose, was to divide the full cell into sections, part going in as a half cell and the remaining section coming out as a full cell, with a slot opened for the rf feed port. This option resulted in a small step of less than $30 \mu \mathrm{m}$ in the full cell, but at a position with lower surface fields. The location of this step was chosen to ensure that it will not become a source of dark currents. The gun was fabricated and the measured dark current data was low as expected. A visual inspection of the inner surface after brazing showed no significant difference, and the $Q$ was sufficiently high. This is a confirmation that the step in the full cell does not exert any negative influences in the cavity parameters. Figure 4 shows the picture of the cells before brazing. Figure 5 shows the complete rf gun after brazing, welding, and tuning. Figure 6 shows the results of field pattern measurements taken by the bead pull method.

The cells were fabricated by starting from a higher frequency and gradually stepping down to the desired frequency and field balance. It took a total of eight cuts of about 10 to $20 \mu \mathrm{m}$ each on the diameter of the cavities to achieve a field balance of 1.0 at a $\pi$ mode frequency of $2856 \mathrm{MHz}$. After brazing, we achieved the 1.0 field balance by using the tuners in the half cell to adjust the frequency downward by about $100 \mathrm{kHz}$. The cells were fabricated from a Hitachi OFHC copper processed by hot isostatic pressing (HIP) [14].

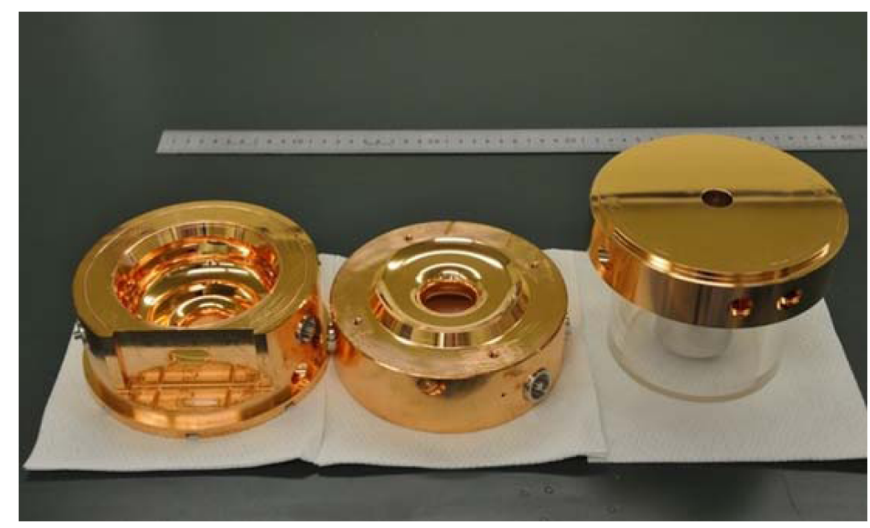

FIG. 4. The rf photocathode gun structure before brazing. Some part of the machined full cell is seen in the center and the other remaining part is in the cavity next to it, at the left in the photograph. The full cell is formed out of this asymmetric cut by brazing. 


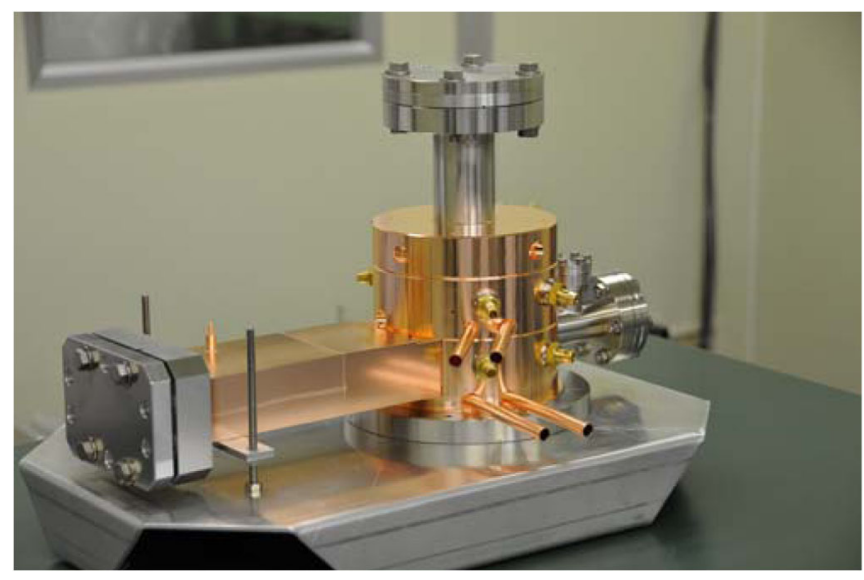

FIG. 5. Complete rf gun after the final tuning. A pair of tuners is positioned just above the water-cooling pipes in each cell. Similar pairs are positioned on the diametrically opposite side.

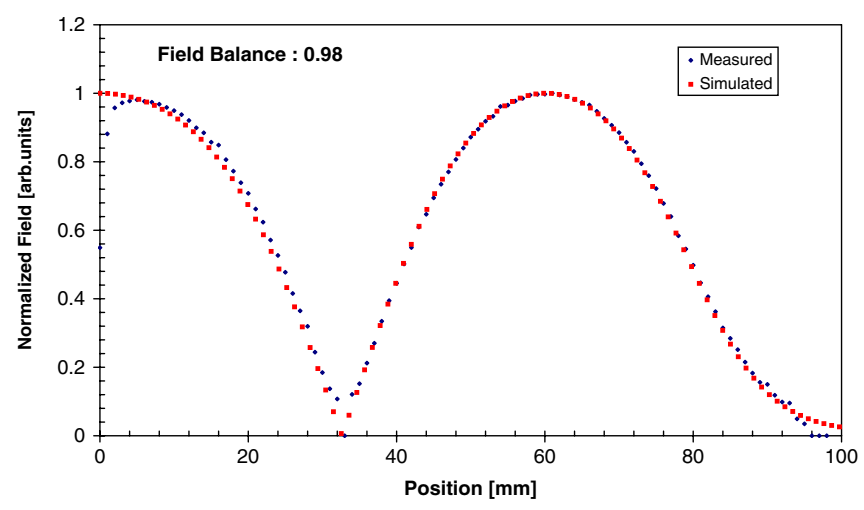

FIG. 6. Measured and simulated field balance for the new gun, after final tuning. The measurements were taken by the bead pull method using a vector network analyzer. The field pattern was measured after each cut on the cavity surface.

Once the rf gun was fully fabricated, we installed it at our facility and carried out rf processing for $150 \mathrm{hrs}$ at a pulse width of $2 \mu \mathrm{s}$ and power of about $10 \mathrm{MW}$. Next, we coated the cathode with cesium-telluride $\left(\mathrm{Cs}_{2} \mathrm{Te}\right)$ and conditioned the gun by operation for another $100 \mathrm{hrs}$. At 30 days after the coating, the quantum efficiency was found to be around $0.5 \%$ and the dark currents were substantially low. At this point we turned on the beam and measured the beam quality. Figure 7 shows the results of the dark current measurement, together with data of the dark currents from the old gun for comparison. The old gun had a quantum efficiency of $\sim 0.3 \%$ at the time of measurement. The plots clearly show that the new gun shows less dark currents than the old gun, even with slightly higher quantum efficiency.

\section{B. Photocathode at LUCX}

The bunch charge in the rf gun depends on the laser power, the choice of photocathode material, and the injection phase. The details of the laser system for the gun have

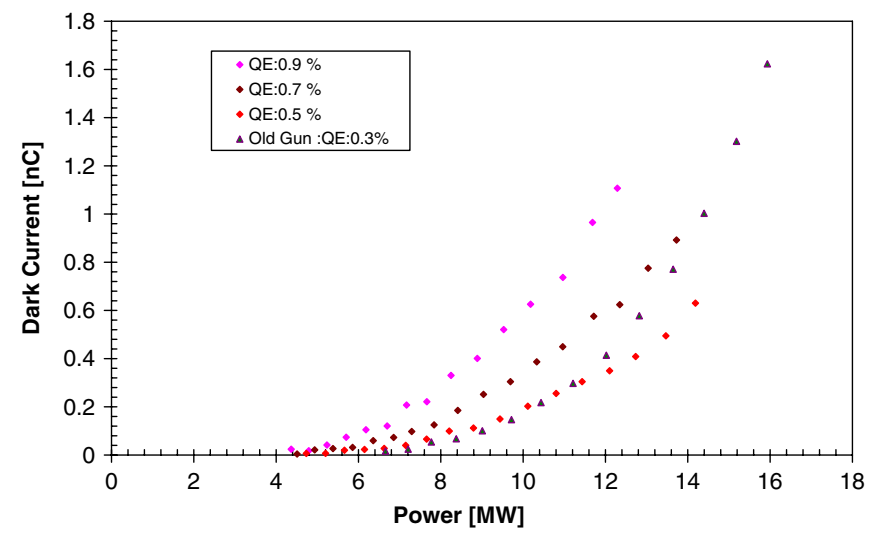

FIG. 7. Measured dark current for the old and new rf guns under similar input power conditions. The new gun shows less dark current than the old gun while maintaining higher quantum efficiency.

been discussed earlier. The injection phase, meanwhile, can be controlled by the user. Because the injection phase also influences the energy spread, we have decided to operate at a phase setting where a minimum energy spread is attained. At LUCX we have achieved stable operation with a $\mathrm{Cs}_{2} \mathrm{Te}$ photocathode technology. The $\mathrm{Cs}_{2} \mathrm{Te}$ has a comparatively short lifetime at the peak quantum efficiency [15]. The quantum efficiency $(\mathrm{QE})$ of $\mathrm{Cs}_{2} \mathrm{Te}$ decreases rapidly over time. In our system, for example, it declined to around $0.5 \%$ in about one month after coating. In our earlier gun, the cathode lasted for as long as about three years with a $\mathrm{QE}$ of about $0.3 \%$ in high fields of about $130 \mathrm{MV} / \mathrm{m}$ [16]. This successful operation in the presence of fields of over $130 \mathrm{MV} / \mathrm{m}$ is a significant achievement and boosts our confidence in the $\mathrm{Cs}_{2} \mathrm{Te}$ photocathode.

Our system uses a molybdenum plug inserted in the rf gun via a load lock mechanism. As an initial conditioning before the $\mathrm{Cs}_{2} \mathrm{Te}$ coating, the plug is inserted at the cathode position and subjected to high fields. The plug is also

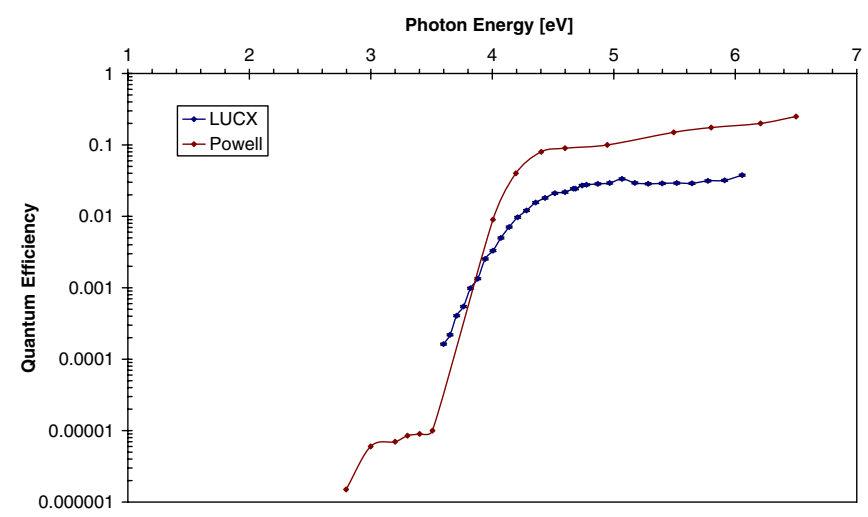

FIG. 8. Measured dependence of quantum efficiency on incident photon energy. The blue curve corresponds to the LUCX data. The brown points correspond to the data from Powell from Ref. [17]. 
cleaned through this conditioning, and at the completion of the process it is transferred to the coating unit using a vacuum chamber. Once the plug is inside the coating chamber, a $10 \mathrm{~nm}$ thick coating of telluride is applied. Then the cesium deposition is commenced, and the quantum efficiency $(\mathrm{QE})$ is monitored over time. The photocurrent peaks at some point, and then starts to fall. In our process, the coating is discontinued when a stable $\mathrm{QE}$ of nearly $3 \%$ is reached. Next, the QE is measured as a function of photon energy. Figure 8 shows the LUCX coating data in blue dots and the data from Powell in brown dots [17]. When we use this photocathode in our gun with our laser system, we easily achieve a charge of around $1 \mathrm{nC}$ per bunch and stable operation with a gradient of $130 \mathrm{MV} / \mathrm{m}$.

\section{BEAM PARAMETER MEASUREMENTS}

The rf gun was carefully tested to find the actual operation parameters at which a low emittance can be achieved. The bunch charge was maintained at $1 \mathrm{nC}$ or more during most of the measurements. Because the energy of the electrons generated from the photocathode is few electron volts, the space-charge forces are strong, resulting in a high emittance beam. To compensate for this degradation, a high gradient of $130 \mathrm{MV} / \mathrm{m}$ is set at the cathode position. Stable operation at this high gradient depends upon good conditioning of the system, as well as a clean cavity surface. A high gradient at the cathode ensures a lowemittance beam with a high bunch charge. In our setup, this high gradient is achieved via careful control of the processing and the maintenance of a good vacuum. A focusing solenoid is introduced at the exit of the rf gun for emittance compensation [18]. In our setup, the 3-meter long, constant-gradient, traveling-wave linac tube accelerates the beam to $50 \mathrm{MeV}$, and a quadrupole doublet after the linac is used to measure the emittance by the quad scan method. Careful tuning is required to ensure that the beam is in good condition before the measurements are commenced. A beam-based alignment (BBA) procedure is applied to ensure that solenoid field variations do not change the beam axis. For a fixed solenoid field, we find the beam position using the beam position monitors (BPM). Then we vary the solenoid field by $\pm 5 \mathrm{~A}$ $(0.012 \mathrm{~T})$ and monitor the variations in the BPM. By changing the laser position at cathode by moving the mirror, we ensure that the variation in BPM reading is less than $10 \mu \mathrm{m}$ for the above field variation.

A highly sensitive alumina fluorescent plate (AF995R, Demarquest Co.) is selected for use as the screen material. The screen is made of $0.5 \%$ chromium oxide doped with alumina and reaches peak scintillation at a wavelength of $693 \mathrm{~nm}$. A 100- $\mu \mathrm{m}$ thick screen is mounted on an inserting device at $45^{\circ}$ relative to the beam. The main disadvantage of this type of screen is a slight enlargement of the scintillation indicating a larger beam size than that of the original beam. This disallows the use of the screen for precise beam size measurements. But with its high sensitivity to even picoampere charge, the screen is very useful for finding beams and monitoring dark currents. Thus, we use the screen mainly for tuning and use an optical transition radiation (OTR) screen for beam size measurements.

One step, in the initial tuning for low emittance, is to minimize the energy spread. After the BBA procedure, the bunch charge variation is determined as a function of the injection phase. The results from this measurement are used to set the injection phase to the minimum energy spread position. In our case, we defined the laser injection phase as zero when the cathode field was zero. Our simulations showed a minimum energy spread at $36^{\circ}$ phase. The injection phase was set accordingly and the beam image was obtained on the screen after the bending magnet. The combined use of this screen and the bending magnet allowed the calibration of the screen for energy variations, thus enabling measurement of the energy spread of the beam. The injection phase was then varied and a final phase was assigned corresponding to the minimum energy spread. Most of our measurements were done at this setting.

After the tuning, the emittance was measured with a quadrupole magnet and the OTR screen located after the collision chamber. The normalized emittance was measured as a function of the solenoid field and a minimum emittance of $1.89 \pm 0.1 \pi \mathrm{mm}$ mrad was observed in the vertical plane. Figure 9 shows the solenoid scan for emittance measurement.

The energy and energy spread were measured at the screen after the bending magnet. Figure 10 shows the variation of the energy spread as a function of the injection phase for a beam with a $1.6 \mathrm{nC}$ charge. As the bunch charge is also a function of the injection phase, the charge is adjusted for each phase position by varying the laser power. For comparison, the figure also plots the measurement results for the old gun with less mode separation. The operating conditions and bunch parameters were the same

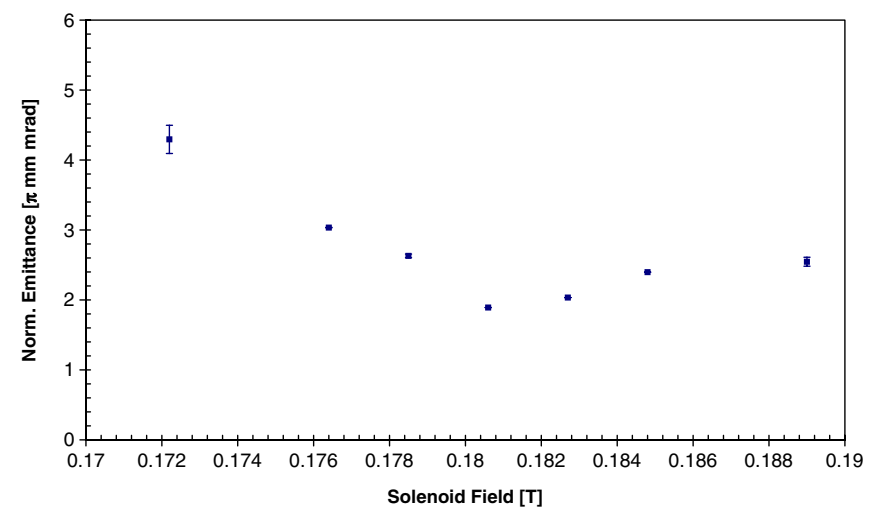

FIG. 9. Solenoid field scan for emittance minimum. The emittance was measured by the quad scan method. The charge per bunch was $1 \mathrm{nC}$. 


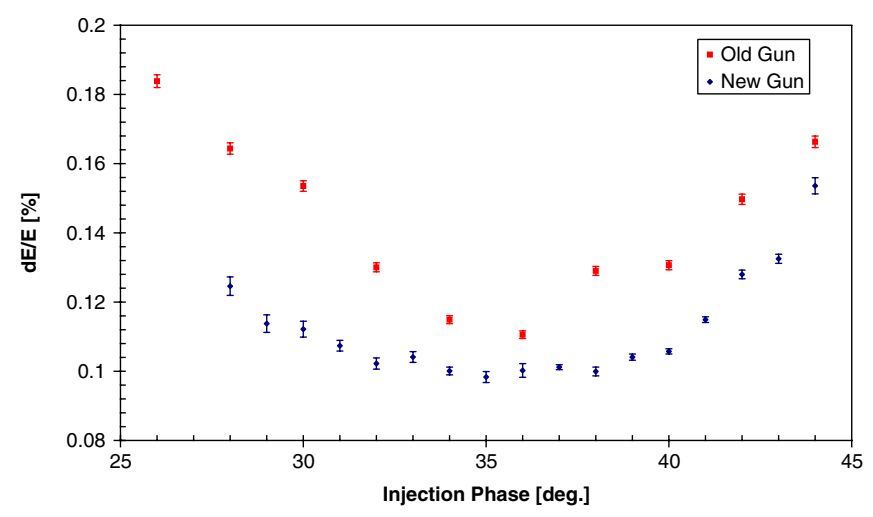

FIG. 10. Energy spread as a function of the injection phase for the old and new guns.

for both measurements. The new gun clearly remains much more stable against the variations of the injection phase.

Simulations by other groups have shown that the energy spread is stable for small variations of the injection phase for systems with larger mode separation [8-10]. Our results demonstrate that an increased mode separation can help maintain a low-energy spread over more phases. This brings additional stability to the operation of the rf gun over environmental parameters and thus increases the reliability of the gun.

\section{MULTIBUNCH BEAM GENERATION}

\section{A. Method of beam-loading compensation}

Our group has worked with a consistent focus on generating a multibunch beam with a lower peak-to-peak energy difference. In earlier experiments using the $\Delta T$ (Delta $T$ ) method for beam-loading compensation with the old gun, we attained a multibunch beam at $40.5 \mathrm{MeV}$ with 100 bunches per train and $0.5 \mathrm{nC}$ charge per bunch [3].

The equation below gives the energy gain with the loading term for the standing-wave rf gun:

$$
\begin{aligned}
V_{\mathrm{RFG}}= & \frac{2 \sqrt{\beta P_{c} Z T^{2}}}{(1+\beta)}\left(1-e^{-t / T_{a}}\right) \\
& -\frac{\omega_{0} Z T^{2} q}{2 Q_{0}}\left[\frac{1-e^{-\left(t-t_{\mathrm{inj}}\right) / T_{a}}}{1-e^{-t_{b} / T_{a}}}+\frac{1}{2}\right],
\end{aligned}
$$

where $\beta$ is the coupling, $P_{c}$ is the input power, $Z T^{2}$ is the effective shunt impedance, $\omega_{0}$ comes from the frequency of the gun, and $Q_{0}$ is the quality factor. $T_{a}$ is the cavity filling time, $t_{\text {inj }}$ is launch time for the first bunch, and $t_{b}$ is the bunch spacing. The loading term depends on the charge per bunch.

From Eq. (1) we can see that the bunch-by-bunch energy for the multibunch beam with a fixed charge and fixed bunch spacing depends on the injection time and input power. A balance between the filling time and injection time should be reached in order to compensate for the bunch-to-bunch energy difference. This method for minimizing the energy spread by varying the injection time is referred to as the " $\Delta T$ (Delta $T$ ) method for beam-loading compensation." For a given injection time, we can increase the power to achieve a good compensation. This is especially useful for attaining good compensation with a high charge and high energy. In theory, a very low peak-to-peak energy difference can be achieved at highenergy levels, such as $50 \mathrm{MeV}$, for high charge bunches, simply by increasing the power and injecting the beam earlier. In practice, however, the achievable level of power has limits and an injection performed too early will result in high single bunch energy spread.

Equation (2) shows the beam energy with loading for a traveling-wave linac:

$$
\begin{aligned}
V_{\mathrm{ACC}}= & \sqrt{P_{c} Z T^{2} L\left(1-e^{-2 \tau}\right)} \\
& -\frac{i_{0} Z T^{2}}{2\left(1-e^{-2 \tau}\right)}\left[1-e^{-2 \tau\left[\left(t-t_{2}\right) / t_{f}\right.}\right] \\
& \left.-2 \tau e^{-2 \tau} \frac{\left(t-t_{2}\right)}{t_{f}}+\tau\left(1-e^{-2 \tau}\right) \frac{t_{b}}{t_{f}}\right]
\end{aligned}
$$

where $t_{f}$ is the filling time, $P_{c}$ is the input power, $Z T^{2}$ is the shunt impedance, $L$ is the length of the linac, $i_{0}$ is the average current, $t_{2}$ is the injection time for the first electron bunch, $t_{b}$ is the bunch spacing, and $\tau$ is an attenuation parameter.

Earlier papers from our group discuss the simulation results for multibunch beam loading [3,5]. Equations (1) and (2) can be used to calculate the various combinations of power, charge, and injection timings for minimizing the peak-to-peak energy differences for long bunch trains. In the next section we present our measurement results and discuss the methods we followed to achieve those results.

\section{B. Experiment No. 1: 100 bunch, $41 \mathrm{MeV}$}

A traveling-wave linac was used for the high-energy acceleration. A pulse compression scheme, the resonant ring compression scheme (RRCS) developed at KEK [19], was applied to satisfy the higher power requirement for compensation of the $50 \mathrm{nC}$ charge in the 100-bunch train. This scheme is very similar to the SLAC energy doubler (SLED), but it uses traveling-wave resonators instead of standing-wave cavities [20,21]. The klystron delivers $4 \mu \mathrm{s}, 40$ MW peak power to the RRCS, resulting in $1 \mu \mathrm{s}, 120 \mathrm{MW}$ peak power after compression. This power is split and delivered to the rf gun and to the linac. With the linac in place and with the power compression scheme on, 100 bunches per train were launched at a $0.5 \mathrm{nC}$ charge per bunch. Then, by varying the injection timing, this $50 \mathrm{nC}$ beam was compensated to attain a minimum peak-to-peak energy variation. Figure 11 shows the measurement results.

Our observations confirmed that if the charge is increased, more power is required to compensate for the beam-loading effect. This is why a high-power klystron 


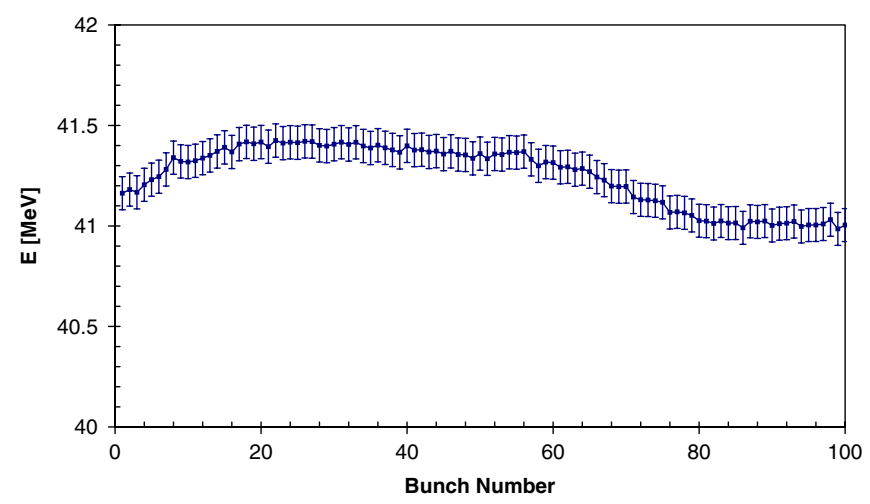

FIG. 11. Beam loading for $41 \mathrm{MeV}, 50 \mathrm{nC}$ in 100 bunches.

must be used when an increase in the bunch charge or number of bunches is required.

In our experiments, we achieved a favorable loading compensation and were able to get a peak-to-peak energy difference of less than $0.7 \%$ with the new gun for $50 \mathrm{nC}$ in 100 bunches. Longer bunch trains are confirmed to lead to additional beam loading, and simulations have shown that they also lead to higher peak-to-peak energy differences. While this rules out the use of the existing linac for long pulse train acceleration, a standing-wave rf gun serves well for the generation of longer bunch trains. This was our reason for replacing the linac with a drift tube [11]. The results of a simulation of the beam performance from the cathode of the gun to the dump with the ASTRA simulation code [22] confirmed that the low-energy beam travels the entire distance to the dump at an approximately 11.5 -meter distance from the cathode, using the same set of magnets. After careful tuning and many trials, the electron bunches were successfully transferred from the gun to the dump with no loss [23].

\section{Experiment No. 2: Low-energy beam with more than 100 bunches}

As mentioned above, the linac was replaced with a long drift tube. Next, through a conditioning of over $50 \mathrm{hrs,}$ vacuum conditions similar to those in experiment No. 1 were regained. The system was then tuned by the methods described earlier, and the beam profile was observed on the OTR screen after the bending magnet. The schematic layout was shown earlier in Fig. 1 and the only difference in low-energy beam generation setup is that the linac is replaced by a long drift tube. A BPM and integrated current transformer (ICT) are placed nearby the screen. Direct measurement of the bunch-by-bunch energy is impossible with our setup. Instead, the energy difference is determined through the use of the BPM, ICT, and OTR screen in combination with the bending magnet. The reading on the ICT is an indication of beam loss, when it occurs. The bending magnet and screen help to measure the single bunch energy, and the BPM registers the orbit for the measured bunch. In the multibunch mode, the average energy and the deviations from the orbit are found with the same BPM. The deviations can be correlated to the energy difference with respect to the average energy, hence the bunch-to-bunch energy difference can be measured. The screen is positioned at a distance of about 10 meters from the rf gun. For a high charge beam, the space-charge forces may result in a blowup of the beam size. Our setup uses the solenoid and quadrupoles to maintain the beam size. If the bunch-to-bunch energy difference is too large, the chicane may reject off some of the bunches. The energy difference, however, can impart a slightly different orbit to the bunches as they pass through the chicane. This results in a positional difference which, if too large, causes some bunches to collide with the wall of the drift chamber. Hence, very careful tuning is needed and the beam loss

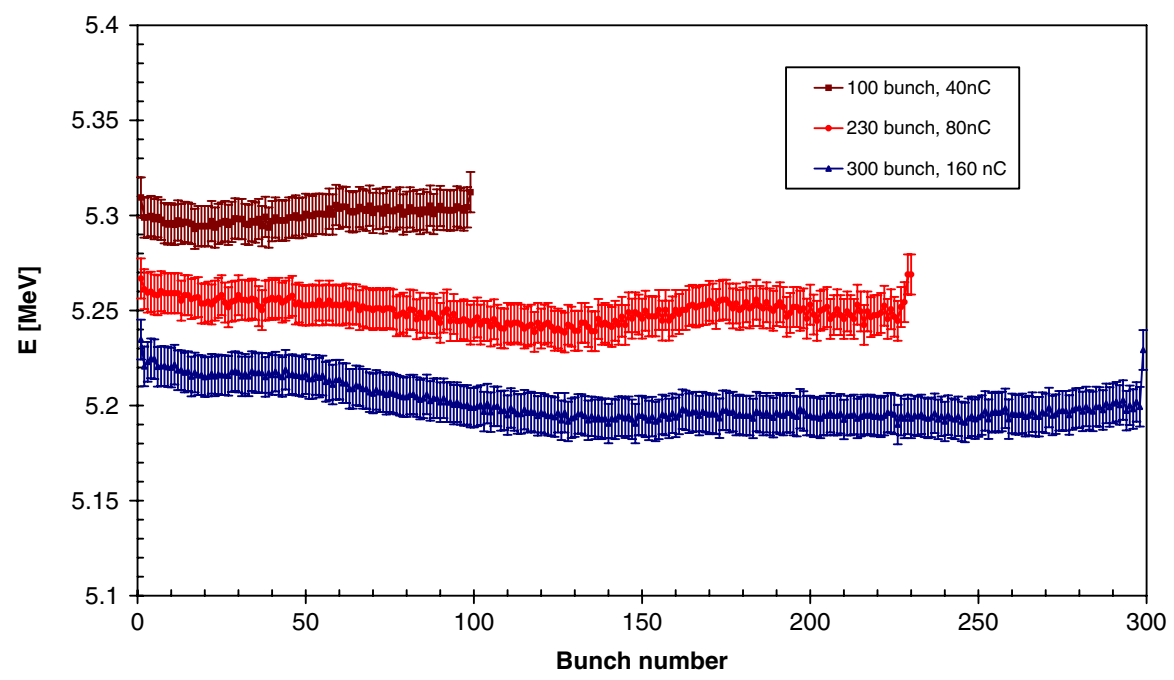

FIG. 12. The top (brown), middle (red), and bottom (blue) curve plot 100-bunch mode at $40 \mathrm{nC}, 230$-bunch mode at $80 \mathrm{nC}$, and 300-bunch mode at $160 \mathrm{nC}$, respectively. 
TABLE II. Measurement results for multibunch beam.

\begin{tabular}{lccc}
\hline \hline Number of bunches & 100 & 100 & 300 \\
Energy [MeV] & 41 & 5.3 & 5.25 \\
Charge per bunch [nC] & 0.5 & 0.5 & 0.55 \\
Peak-to-peak energy difference [\%] & 0.7 & 0.36 & 0.85 \\
\hline \hline
\end{tabular}

must be monitored to ensure that none occurs across the drift tube.

Upon completing these initial settings, we planned an experiment for a 300-bunch beam. We found, at this point, that the Pockels cell for the laser was capable of generating 300 bunches. Above 300 bunches, the Pockels cell waveform begins to ring and thus becomes unusable. The beam was carefully tuned, starting from 4-bunch mode and gradually proceeding upwards to $36,100,150,230$, and finally 300 bunches. The system was carefully tuned at each stage to ensure that the beam went to the dump. Next, the bunch charge was gradually increased to $0.55 \mathrm{nC}$ per bunch. Figure 12 shows the results for 100-, 230-, and 300bunch mode operation. The 100-bunch mode was performed at a charge of a $40 \mathrm{nC}$ charge; the 230-bunch mode, at $80 \mathrm{nC}$; and the 300 -bunch mode, at $160 \mathrm{nC}$.

As the bunch charge increased, the beam clearly had to be injected earlier to ensure good compensation. This resulted in a low-energy beam. To get a similar level of energy in each case, more power will be needed into the rf gun. As seen from the above plot, 300 bunches per train were successfully generated at $5 \mathrm{MeV}$ with a $160 \mathrm{nC}$ total charge. The peak-to-peak energy difference was $0.85 \%$ for this 300-bunch train. The peak-to-peak energy difference continued to rise as the bunch number increased. Table II below shows the comparative results for multibunch beam generation. Figure 13 compares the calculated and measured values for the same input conditions.

This setup successfully generated a long bunch train with high charge and a low-energy difference. The Pockels cell had to be changed in order to attain longer pulse trains from the laser. Upon completing a planned upgrade for the laser system, we plan to achieve 8000

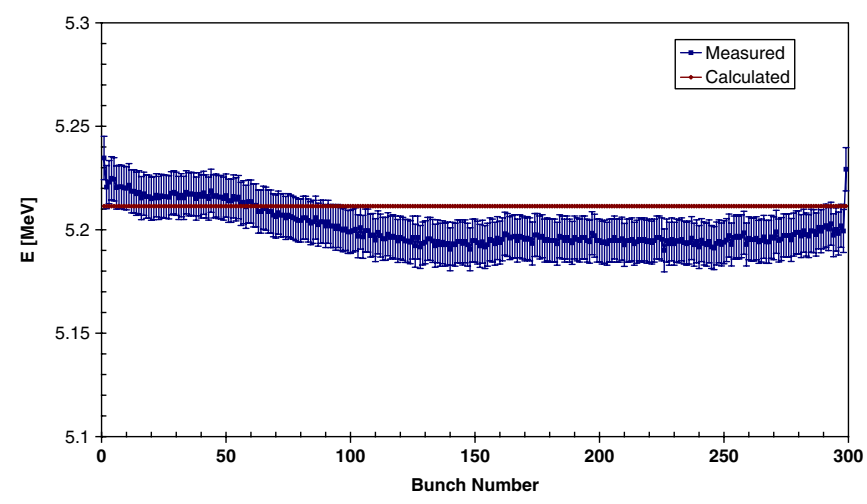

FIG. 13. 300 bunches, total charge generation of $160 \mathrm{nC}$, and comparison with calculation. bunches at $5 \mathrm{MeV}$ with a $0.5 \mathrm{nC}$ charge per bunch [2]. The recent results obtained by PHIN gun are for similar high charge accelerations and are very encouraging [24]. The important thing in our setup is that we transport the bunches to a long distance of about $10 \mathrm{~m}$ with a low peakto-peak energy difference. For the high-energy case, our target is to achieve 100 bunches with a $2 \mathrm{nC}$ charge per bunch. A high-power, long-pulse klystron will be required for both trials. To meet this condition, we will be upgrading to an $\mathrm{rf}$ power system with a separate klystron with a peak power of $24 \mathrm{MW}$ and a pulse width of $24 \mu \mathrm{s}$ for the rf gun. The existing 40 MW klystron with the RRCS will then drive the linac. This will be possible by the end of this year.

\section{DISCUSSIONS AND REMARKS}

Our group has designed and successfully commissioned a larger-mode-separation rf gun at the LUCX facility at KEK. We use a $\mathrm{Cs}_{2} \mathrm{Te}$ photocathode and successfully operate the rf gun at a high gradient of $130 \mathrm{MV} / \mathrm{m}$ at the cathode. This is one of the most interesting features of our rf gun activity. We increased the separation between $\pi$ and zero mode to $8.67 \mathrm{MHz}$ and tuned the gun to a field balance of 1.0. The measured normalized transverse emittance was around $1.89 \pm 0.1 \pi \mathrm{mm}$ mrad. We measured the energy spread as a function of the injection phase and compared the same with the data from the old gun with $3.5 \mathrm{MHz}$ mode separation. The large-mode-separation gun had less energy spread variations over large phase variations. This is the first observation of this kind and confirms the need for large-mode-separation rf guns. The higher mode separation will increase the stability of the gun, help to produce good-quality beams over long runs, and become an essential precondition for long-pulse-width rf power.

A multibunch beam with lower peak-to-peak energy differences will be very crucial for inverse Compton sources. It will boost both the charge delivered per second and the x-ray flux per second. Our group proposes the launch of an 8000-bunch train with an $0.5 \mathrm{nC}$ charge per bunch. This high charge per train will be focused in the collision chamber at about $8 \mathrm{~m}$ from the cathode. This paper establishes the basic research work towards this goal. In our current experiments, we successfully accelerated 100 bunches with a $50 \mathrm{nC}$ charge at $41 \mathrm{MeV}$ with a peak-to-peak energy difference of less than $0.7 \%$. We then accelerated 300 bunches with a $0.55 \mathrm{nC}$ charge per bunch (160 $\mathrm{nC}$ charge) at $5 \mathrm{MeV}$ with a peak-to-peak energy difference of less than $0.85 \%$. This was one of our most important results.

The acceleration of a longer train is still restricted by the limitations of the Pockels cell. Replacement of the Pockels cell will help us achieve longer pulse trains. Then, our next step will be to replace the klystron and rf power delivery system to input $24 \mathrm{MW}, 24 \mu \mathrm{s}$ rf power in the gun.

We have started building a 3.5 cell rf gun as an extension of the newly designed rf gun reported here. This will help 
us achieve energy of around $10 \mathrm{MeV}$ at the gun exit. In the next stage we plan to build a compact, high gradient linac to achieve a $30 \mathrm{MeV}$ beam with 8000 bunches. The entire accelerating system will be small enough to fit on a 3-meter-long, 1-meter-wide table.

\section{ACKNOWLEDGMENTS}

We are thankful to the staff members of ATF, KEK for their constant support of this activity. Mr. Takatomi and Mr. Kudo also provided valuable support for the fabrication and the brazing. We thank Dr. Omori for his active follow-up on the multibunch generation and to Dr. Y. Honda and Dr. A. Aryshev for their active discussions. This work was funded by a grant-in-aid from JSPS (KAKENHI 17GS0210) under the Ministry of Education, Science and Technology (MEXT) of Japan. Additional support was provided under the Quantum Beam Technology Program of MEXT. One of the authors (Abhay Deshpande) was supported by a MEXT scholarship for foreign research students.

[1] K. Hirano et al., Nucl. Instrum. Methods Phys. Res., Sect. A 560, 233 (2006).

[2] M. Fukuda et al., Nucl. Instrum. Methods Phys. Res., Sect. A 637, S67 (2011).

[3] S. Liu et al., Nucl. Instrum. Methods Phys. Res., Sect. A 584, 1 (2008).

[4] K. Sakaue et al., Rev. Sci. Instrum. 80, 123304 (2009).

[5] Abhay Deshpande et al., Nucl. Instrum. Methods Phys. Res., Sect. A 600, 361 (2009).

[6] D. H. Dowell et al., Report No. SLAC-PUB-13401, 2008.

[7] R. Akre et al., Phys. Rev. ST Accel. Beams 11, 030703 (2008).

[8] C. Limborg et al., LCLS Technical Note, Report No. LCLS-TN-05-3, 2005.
[9] J. Y. Raguin et al., in Proceedings of Free Electron Laser, Stanford, USA, 2005, JaCOW eConf C0508213 (SLAC, California, 2005), pp. 324-27 [http://www.slac.stanford .edu/econf/C0508213/PAPERS/TUPP048.PDF].

[10] D. H. Dowell et al., Nucl. Instrum. Methods Phys. Res., Sect. A 528, 316 (2004).

[11] Abhay Deshpande et al., in Proceedings of the 23rd Particle Accelerator Conference, Vancouver, Canada, 2009 (IEEE, Piscataway, NJ, 2009), MO6RFP065, pp. 515-517 [http://epaper.kek.jp/PAC2009/papers/ mo6rfp065.pdf].

[12] Y. Kamiya et al., in Proceedings of the 2007 Particle Accelerator Conference, Albuquerque, New Mexico (IEEE, New York, 2007), THPMN040, pp. 2808-2810 [http://epaper.kek.jp/p07/PAPERS/THPMN040.PDF].

[13] K. Halbach and R.F. Holsinger, Part. Accel. 7, 213 (1976) [http://cdsweb.cern.ch/record/700607].

[14] H. Matsumoto et al., KEK Report No. 91-47, 1991 [http:// cdsweb.cern.ch/record/224889].

[15] S. H. Kong et al., J. Appl. Phys. 77, 6031 (1995).

[16] K. Sakaue, Ph.D. thesis, Waseda University, 2009.

[17] R. A. Powell et al., Phys. Rev. B 8, 3987 (1973).

[18] B. E. Carlsten, Nucl. Instrum. Methods Phys. Res., Sect. A 285, 313 (1989).

[19] S. Yamaguchi et al., KEK Report No. 94-87, 1994 [http:// cdsweb.cern.ch/record/275152/].

[20] Z. D. Farkas, Report No. SLAC-TN-73-08, 1973.

[21] P. B. Wilson, Report No. SLAC-TN-73-15, 1975.

[22] K. Flöttmann, A Space Charge Tracking Algorithm, ASTRA, http://www.desy.de/ mpyflo/.

[23] Abhay Deshpande et al., in Proceedings of International Particle Accelerator Conference, Kyoto, Japan, 2010 (IPAC'10/AFCA, Kyoto, Japan, 2010), THEPC026, pp. 4104-4106 [http://epaper.kek.jp/IPAC10/papers/ thpec026.pdf].

[24] M. Petrarca et al., in Proceedings of International Particle Accelerator Conference, Kyoto, Japan, 2010 (Ref. [23]), THEPC032, pp. 4122-4124 [http://epaper.kek.jp/IPAC10/ papers/thpec032.pdf]. 